\title{
PD-L1 expression correlation with metabolic parameters of FDG PET/CT and clinicopathological characteristics in non- small cell lung cancer
}

\author{
Xiaodong $\mathrm{Wu}^{1,2}$, Yan Huang ${ }^{3}$, Qingping Zhao ${ }^{1}$, Lei Wang ${ }^{4}$, Xiao Song ${ }^{5}$, Yi Li $^{1}$ and Lei Jiang ${ }^{1 *}$ (D)
}

\begin{abstract}
Background: Immunotherapy targeting programmed cell death 1 (PD-1) or its ligand 1 (PD-L1) has shown promising results in non-small cell lung cancer (NSCLC) patients. Exploring PD-L1 expression could help to select NSCLC candidates for immunotherapy. Fluorine-18 fluorodeoxyglucose (FDG) PET/CT could provide phenotypic information on malignant tumors. Thus, this study investigated PD-L1 expression correlation with metabolic parameters of FDG PET/CT and clinicopathological characteristics in NSCLC.

Methods: FDG PET/CT metabolic parameters including maximum standard uptake (SUVmax), metabolic tumor volume and total lesion glycolysis of primary lesion (MTV-P, TLG-P), and combination of primary lesion and metastases (MTV-C, TLG-C) were compared with PD-L1-positive expression in patients with NSCLC. Moreover, clinicopathological characteristics, including age, gender, smoking history, serum tumor markers, tumor location, size, TNM stage, and genetic mutation were also reviewed.

Results: All 374 patients (215 men; 159 women; age $63 \pm 9$ years) included 283 adenocarcinomas (ACs) and 91 squamous cell carcinomas (SCCS). PD-L1 expression was positive in 27.8\% (104/374) cases. SUVmax, TLG-P, and TLG-C of PD-L1 positivity were significantly higher than PD-L1 negativity. Moreover, PD-L1 expression was obviously correlated with man, smoking, and central NSCLC. If ACs and SCCs were separately analyzed, PD-L1 positivity in ACs and SCCs was 21.6\% (61/283) and 47.5\% (43/91), respectively, and only SUVmax was obviously associated with PD-L1 expression. Furthermore, multivariate analysis revealed that only SUVmax was an independent predictor of PD-L1 positive expression in overall NSCLC, AC, and SCC. Using a SUVmax cut-off value of 12.5, PD-L1 status of NSCLC was predicted by FDG PET/CT with sensitivity, specificity, and accuracy of $65.4 \%, 86.7 \%$, and $80.7 \%$, respectively.

Conclusions: PD-L1 expression of NSCLC was related to SUVmax, TLG, man, smoking, and central location. However, only SUVmax was an independent predictor of PD-L1 positivity, which could help to explore the existence of immune checkpoints.
\end{abstract}

Keywords: FDG, PET/CT, PD-L1, Non-small cell lung cancer

\footnotetext{
* Correspondence: leijiang1031@163.com

${ }^{1}$ Department of Nuclear Medicine, Shanghai Pulmonary Hospital, Tongji

University School of Medicine, 507 Zhengmin Road, Shanghai 200433, China

Full list of author information is available at the end of the article
}

\section{Springer Open}

(c) The Author(s). 2020 Open Access This article is licensed under a Creative Commons Attribution 4.0 International License, which permits use, sharing, adaptation, distribution and reproduction in any medium or format, as long as you give appropriate credit to the original author(s) and the source, provide a link to the Creative Commons licence, and indicate if changes were made. The images or other third party material in this article are included in the article's Creative Commons licence, unless indicated otherwise in a credit line to the material. If material is not included in the article's Creative Commons licence and your intended use is not permitted by statutory regulation or exceeds the permitted use, you will need to obtain permission directly from the copyright holder. To view a copy of this licence, visit http://creativecommons.org/licenses/by/4.0/. 


\section{Background}

Lung cancer is the most common cancer and the leading cause of cancer death all over the world [1], and non-small cell lung cancer (NSCLC) is the most commonly diagnosed histological subtype [2-4]. Despite the development in treatment for NSCLC such as thoracoscopic surgery, chemotherapy, radiotherapy, and targeted therapy $[5,6]$, the overall 5-year survival rate is still poor [7]. According to the data from 2009 to 2015, the 5-year survival rate of patients with lung cancer is as low as $19.4 \%$, while that of patients with distant metastasis is only 5.2\% [8]. Nowadays, many studies indicated that immunotherapy targeting programmed cell death ligand-1 (PD-L1) could provide a promising new way for the treatment of NSCLC $[9,10]$. Antibodies blocking PD-1/PD-L1 pathway can enhance anti-tumor immunity to achieve the goal of killing cancer cells [11]. Thus, it is necessary to explore the existence of immune checkpoints and to select NSCLC candidates for immunotherapy.

At present, immunohistochemistry is the main method for identifying tumor PD-L1 expression, which requires surgical or biopsied tumor specimens from patients with NSCLC [12]. However, these procedures such as surgery, transbronchial lung biopsy, or CT-guided biopsy are invasive and even failed due to the patients' physical condition or unqualified specimens. Fluorine-18 fluorodeoxyglucose (FDG) PET/CT is a noninvasive imaging modality and can be applied even if specimens from patients are not available, which has been widely applied to predict many molecular phenotypes of malignant tumors, such as histological types, tumor differentiation, proliferation, hypoxia, and genetic mutation [13-17].

The previous studies have confirmed that tumor expression of PD-L1 was related to high glucose metabolism of PET/CT in NSCLC [18-22]. However, almost all previous studies focused on the correlation of the FDG PET/CT metabolic parameter of maximum standard uptake (SUVmax) with PD-L1 expression [18-22]. SUVmax is easy to determine and reflects the maximum uptake of glucose by metabolically active lesions. Volume-based PET parameters such as metabolic tumor volume (MTV) and total lesion glycolysis (TLG) represent glucose activity in the entire tumor mass, which could also reflect the metabolic status of the malignant tumors [23]. However, at present, little is known about the association of PD-L1 with MTV and TLG.

Therefore, in this study, FDG PET/CT findings and metabolic parameters of SUVmax, MTV and TLG of primary lesion (MTV-P and TLG-P), and combination of primary lesion and metastases (MTV-C and TLG-C) were retrospectively analyzed in 374 patients with NSCLC. We investigated tumor PD-L1 positive expression correlation with these metabolic parameters and patients' clinicopathological characteristics and analyzed their roles in predicting PD-L1 expression in NSCLC.

\section{Materials and methods \\ Patients}

From January 2017 to August 2018, 374 NSCLC patients with definite PD-L1 expression results who underwent FDG PET/CT examination in our department were included in this study according to the following criteria: (1) the interval between PET/CT examination and pathological diagnosis was no more than 2 weeks; (2) only adenocarcinoma (AC) and squamous cell carcinoma (SCC) were included, and other NSCLC subtypes were excluded; (3) there was no other cancer history or coexisting other malignant tumors; and (4) the patients did not receive any prior systemic or local tumor therapy.

\section{FDG PET/CT scan}

These scans were performed on a Biograph 64 system (Siemens Healthineers, Erlangen, Germany) with a 21.6 $\mathrm{cm}$ axial field of view. The patients were required to fast for at least $6 \mathrm{~h}$ prior to imaging, and serum glucose levels were kept lower than $7.4 \mathrm{mmol} / \mathrm{l}$. Images were captured $\sim 60 \mathrm{~min}$ after intravenous administration of 3.7 MBq of FDG per kilogram of body weight. Six or 7 bed positions from the base of the skull to the midthighs were imaged. PET images were acquired for 2.5 min per bed position. CT was performed on the same scanner without contrast administration, and CT scan data were collected under the following conditions: 120 $\mathrm{kV}, 101 \mathrm{~mA}$ (adjusted by auto $\mathrm{mA}$ ), and a gantry rotation speed of $0.5 \mathrm{~s}$. All the CT scans were conducted via 5-mm-thick axial slices. PET images were reconstructed at $200 \times 200$ pixels using a Gaussian filter of $5.0 \mathrm{~mm}$ full width at half maximum value. All image reconstructions were performed with the ordered-subset expectationmaximization algorithm, incorporating a CT-based transmission map.

Regions of interest (ROIs) were defined around the pulmonary lesions, and the maximum and mean values of an ROI were defined as the SUVmax and SUVmean, respectively. MTV-P and MTV-C were computed with $40 \%$ of SUVmax as a threshold. TLG-P and TLG-C were calculated according to the following formula: TLG = SUVmean $\times$ MTV [22, 24]. PET/CT imaging results were analyzed and interpreted by 2 experienced nuclear medicine physicians who were unaware of the patients' clinical information, other conventional imaging findings, and pathology results.

\section{Histopathological and genetic analyses}

Tumor specimens in this study were obtained after surgical excision or biopsy. The specimens were carefully examined, and the part with more malignant cells, less differentiated cells, and less hemorrhage and necrosis were subjected to histopathological quantification of PDL1 expression. The platform of Dako Link 48 and the 
antibody of Dako 22C3 were used for PD-L1 staining. Tumor proportion score (TPS) was recorded as the percentage of PD-L1 positive tumor cells over all tumor cells, and TPS $\geq 1 \%$ were considered PD-L1 positive expression [22, 25].

Moreover, we recorded and counted gene mutations in patients who had undergone genetic testing, including epidermal growth factor receptor $(E G F R)$, Kirsten rat sarcoma viral oncogene homolog gene (KRAS), echinoderm microtubule-associated protein-like 4-anaplastic lymphoma kinase fusion gene (EML4-ALK), v-Raf murine sarcoma viral oncogene homolog $\mathrm{B} 1$ (BRAF) proto-oncogene, and c-Ros oncogene 1 (ROS1). These histopathological and genetic results were reviewed by 2 experienced pathologists.

\section{Statistical analysis}

Data are expressed as the mean \pm SD. Univariate analysis of differences between groups was determined using the independent $t$ test, one-way ANOVA, or chisquared test, where applicable. Multivariate analysis of the relationship between PD-L1 expression and selected factors which have significance via univariate analysis was performed by logistic regression. The receiver operating curve was performed to determine the optimal SUVmax cut-off value using bootstrapping of $R$ package for predicting the expression of PD-L1, and random sampling was conducted 1000 times from all 374 cases. A $P$ value $<0.05$ was considered statistically significant. SPSS 21.0 software for Windows (IBM Corp., Armonk, NY, USA) was used for the statistical analysis.

\section{Results}

\section{Patients' clinicopathological characteristics}

All 374 patients included 215 men and 159 women, with an average age of $63 \pm 9$ years (range 32-87 years). Smoking history was found in 205 patients and all of them were men. A total of 359 out of 374 patients were examined for serum tumor markers, and 73 had elevated levels of cytokeratin 211 (CYFRA211, reference range < $3.3 \mathrm{ng} / \mathrm{ml}$ ), 40 had elevated carcinoembryonic antigen (CEA, $<10 \mu \mathrm{g} / \mathrm{l}), 8$ had elevated neuron-specific enolase $(\mathrm{NSE},<20 \mathrm{ng} / \mathrm{ml}$ ), and 1 had elevated pro-gastrinreleasing peptide (proGRP, $<3 \mathrm{ng} / \mathrm{ml}$ ), respectively. No patient was found to have elevated squamous cell carcinoma antigen (SCCA, $<3 \mathrm{ng} / \mathrm{ml}$ ).

All patients presented with solitary primary lesion with a mean diameter of $32 \pm 15 \mathrm{~mm}$ (range 7-120 mm), including 283 ACs and 91 SCCs. Fourteen cases were central tumors and 360 were peripheral. The TNM stages of 374 cases were as follows: 235 cases were at stage I, 77 at stage II, 60 at stage III, and 2 at stage IV. PD-L1 positive expression was detected in 104 of 374 cases, and the positive rate was $27.8 \%$. A total of 349 out of 374 patients underwent genetic testing, EGFR mutation was found in 145 patients, KRAS mutation in 26, EML4-ALK fusion in 5 , and ROS1 mutation in 2, respectively. No $B R A F$ mutation was observed.

\section{PET/CT}

FDG uptake was avid in all 374 primary lung tumors with the average SUVmax, MTV-P, and TLG-P of $9.5 \pm$ 5.5 (1.1-24.5), $13.3 \pm 19.1$ (0.7-168.9), and $96.8 \pm 192.2$ (1.5-1457.1), respectively. Among 374 patients, 88 cases had local and distant metastases, including 86 with only lymph node metastases, 1 coexistence with lymph node and pleural metastases, and other one coexistence with lymph node and adrenal metastases. Therefore, the mean MTV-C and TLG-C of all cases were $14.1 \pm 19.5$ (0.7-168.9) and $99.3 \pm 193.9$ (1.5-1457.1), respectively.

\section{PD-L1 correlation with PET/CT and clinicopathological features in NSCLC}

SUVmax, TLG-P, and TLG-C in PD-L1 positive tumors were significantly higher than those in PD-L1 negative tumors $(P<0.05)$ (Table 1, Fig. 1$)$. And no significant differences in MTV-P and MTV-C were found between PD-L1 positive and negative groups $(P>0.05$, Table 1$)$. There was an obvious correlation between PD-L1 expression and man, smoking, and central NSCLC $(P<$ 0.05 , Table 1). No significant differences in other clinicopathological features such as age, serum tumor markers, tumor size, TNM stage, and genetic mutation were found between PD-L1 positive and negative groups $(P>$ 0.05, Table 1).

Multivariate analysis showed that only SUVmax was an independent predictor of PD-L1 expression in NSCLC (Table 2, Fig. 2). Furthermore, the optimal SUVmax threshold to predict PD-L1 expression of NSCLC was investigated. The best SUVmax cut-off value was determined to be 12.5 using bootstrapping analysis and the area under the curve was 0.845 (95\% CI $0.802-$ 0.888 ). PD-L1 status of NSCLC could be predicted by SUVmax with sensitivity, specificity, and accuracy of $65.4 \%, 86.7 \%$, and $80.7 \%$, respectively (Fig. 3a).

\section{PD-L1 correlation with PET/CT and clinicopathological features in pathological subgroups}

The percentage of PD-L1 positive expression was $21.6 \%$ $(61 / 283)$ and $47.3 \%$ (43/91) in AC and SCC, respectively. PD-L1 positive cases were significantly higher in SCC than in $\mathrm{AC}(P<0.05)$. For both $\mathrm{AC}$ and SCC subgroups, SUVmax in PD-L1 positive tumors was significantly higher than that in PD-L1 negative tumors $(P<0.05)$ (Table 1, Fig. 4). And no significant differences in MTV, TLG, and clinicopathological features between PD-L1 positive and negative groups were noted $(P>0.05$, Table 1$)$. 


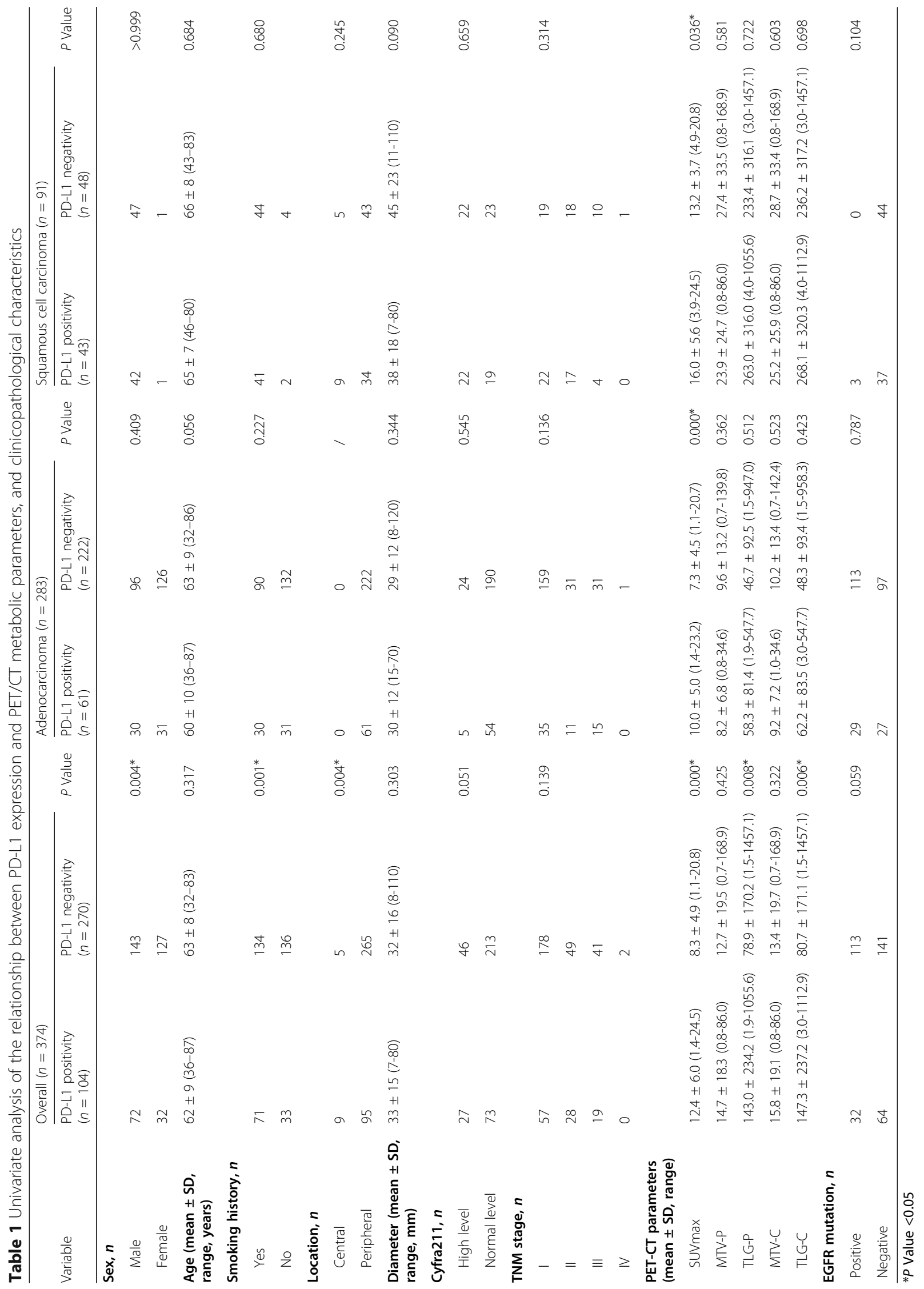



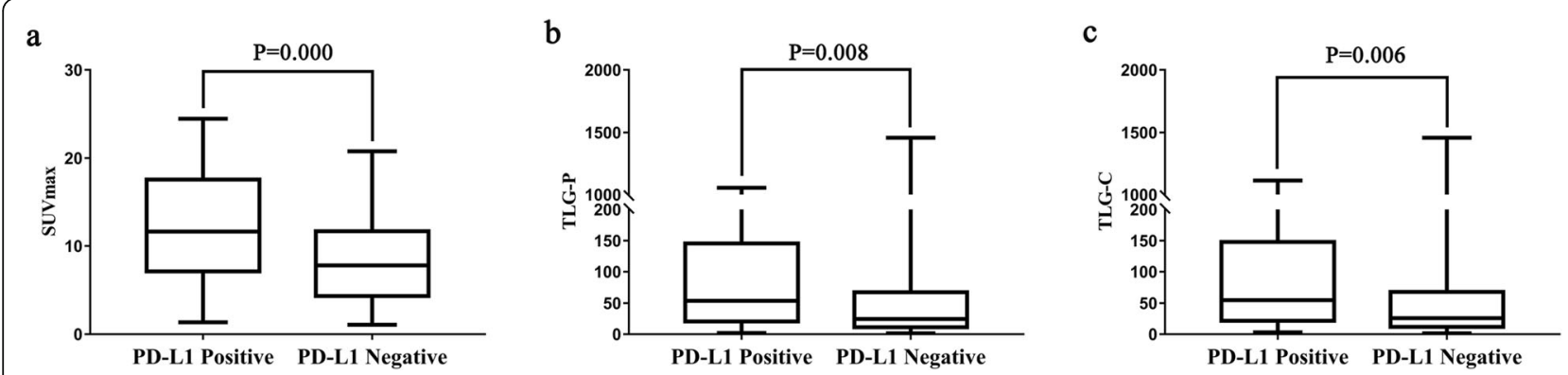

Fig. 1 The correlation between PD-L1 expression and SUVmax (a), TLG-P (b), and TLG-C (c) in overall NSCLC

Multivariate analysis also revealed that SUVmax was an independent predictor of PD-L1 positive expression in both AC and SCC subgroups (Table 2). Based on bootstrapping analysis, the best SUVmax cut-off value was determined to be 7.2 and 16.3 in AC and SCC subgroups, respectively, and the area under the curve was 0.832 (95\% CI $0.780-0.884$ ) and 0.848 (95\% CI $0.763-$ 0.933), respectively. PD-L1 status of lung AC and SCC could be predicted by FDG PET/CT with sensitivity, specificity, and accuracy of $83.6 \%$ vs $69.8 \%, 60.8 \%$ vs $89.6 \%$, and $65.7 \%$ vs $71.4 \%$, respectively (Fig. $3 \mathrm{~b}$ and c).

\section{Discussion}

In recent years, immunotherapy targeting PD-L1 has shown promising results in NSCLC patients. Different TPS values were used in previous studies for evaluating tumor PD-L1 expression, including 1\%, 5\%, 10\%, and
$50 \%[26,27]$. The latest version of the National Comprehensive Cancer Network reported NSCLC patients could benefit from immunotherapy when TPS was over 1\% [12]. For example, when TPS $\geq 50 \%$, PD-1/PD-L1 antibody combined with chemotherapy is the best choice for ACC, and PD-1/PD-L1 antibody alone is the best choice for SCC. When TPS during $1 \%$ to $49 \%$, PD-1/PD-L1 antibody combined with chemotherapy is prior to PD-1/ PD-L1 antibody alone for both ACC and SCC. When TPS $<1 \%$, PD-1/PD-L1 antibody combined with chemotherapy is better than chemotherapy alone for both ACC and SCC [28]. Therefore, we chose TPS $\geq 1 \%$ as PD-L1 positive in this study and investigated PD-L1 expression correlation with metabolic parameters of FDG PET/CT and clinicopathological characteristics in NSCLC.

Several reports suggested that the association between the percentages of PD-L1 positive tumors and histological

Table 2 Multivariate analysis of the relationship between PD-L1 expression and selected factors which have significance via univariate analysis by logistic regression SUVmax as an independent predictor of PD-L1 expression by multivariate analysis

\begin{tabular}{|c|c|c|c|c|}
\hline & Factor & Odds ratio & 95\% confidence interval & $P$ value \\
\hline \multirow[t]{6}{*}{ Overall $(n=374)$} & Sex & 0.38 & $0.07-2.11$ & 0.268 \\
\hline & Smoking & 3.86 & $0.70-21.18$ & 0.121 \\
\hline & Location & 2.55 & $0.757-8.582$ & 0.131 \\
\hline & SUVmax & 1.14 & $1.08-1.20$ & $0.000^{*}$ \\
\hline & TLG-P & 0.98 & $0.96-1.01$ & 0.109 \\
\hline & TLG-C & 1.02 & $0.99-1.05$ & 0.129 \\
\hline \multirow[t]{5}{*}{$\mathrm{AC}(n=283)$} & Sex & 0.28 & $0.02-3.33$ & 0.313 \\
\hline & Smoking & 4.70 & $0.39-55.97$ & 0.221 \\
\hline & SUVmax & 1.16 & $1.07-1.24$ & $0.000^{*}$ \\
\hline & TLG-P & 0.97 & $0.93-1.01$ & 0.104 \\
\hline & TLG-C & 1.03 & $0.99-1.07$ & 0.147 \\
\hline \multirow[t]{6}{*}{$\operatorname{SCC}(n=91)$} & Sex & 0.18 & $0.01-7.21$ & 0.358 \\
\hline & Smoking & 3.40 & $0.30-38.09$ & 0.320 \\
\hline & Location & 1.97 & $0.57-6.79$ & 0.281 \\
\hline & SUVmax & 1.12 & $1.01-1.24$ & $0.032^{*}$ \\
\hline & TLG-P & 0.99 & $0.95-1.02$ & 0.381 \\
\hline & TLG-C & 1.01 & $0.98-1.05$ & 0.407 \\
\hline
\end{tabular}




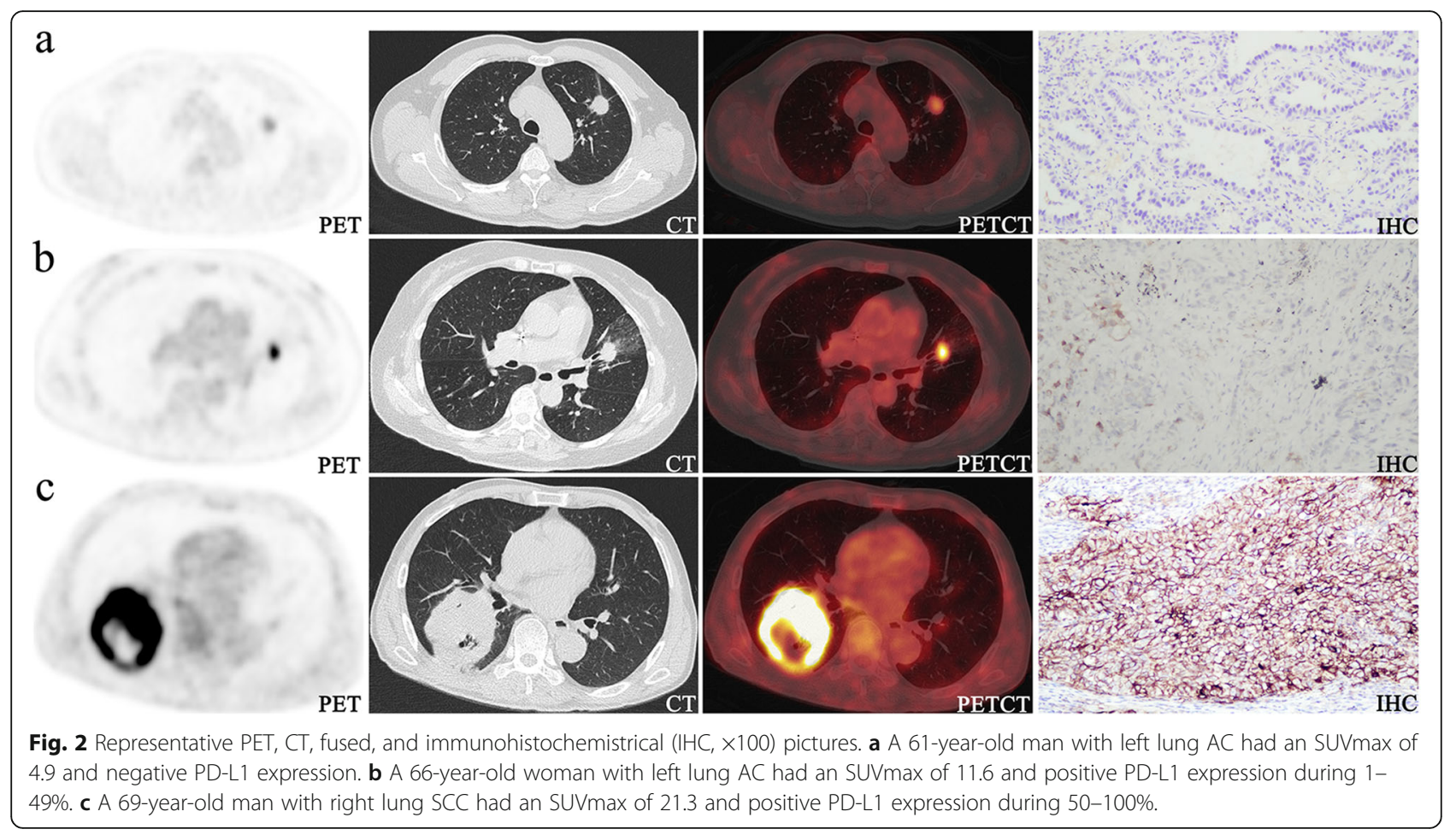

subtypes of NSCLC was found although the criteria of PD-L1 positivity varied in each study. Janzic et al. [26] reported that PD-L1 positive (TPS $\geq 5 \%$ ) cases were higher in SCC (52\%) than in AC (17\%). Lin et al. [27] showed that PD-L1 positivity (TPS $\geq 1 \%$ ) was more frequently observed in SCC (46\%) than in AC (27\%). Miyazawa et al. [29] compared the percentages of PD-L1 positive NSCLCs using the same criteria as Lin et al. [27], which were confirmed to be more frequent in SCC (44\%) or large cell carcinoma (67\%) than in AC (21\%). In this study, the percentage of tumors with PD-L1 positive expression was $27.8 \%, 21.6 \%$, and $47.3 \%$ in overall NSCLC, AC, and SCC, respectively. PD-L1 positive rate of SCC was significantly higher than that of $\mathrm{AC}$, which was consistent with the previous reports [26, 30]. Moreover, our previous study [22] demonstrated that the positive rate of PD-L1 expression in pulmonary sarcomatoid carcinoma (PSC) was $87.5 \%$, which was higher than that of SCC and AC in this study under the same criteria.

At present, some studies have demonstrated that the predictive value of SUVmax on FDG PET/CT in PD-L1 expression from the primary tumor in patients with lung cancer at the initial diagnosis. For example, Kaira et al. [21] demonstrated that PD-L1 expression level was significantly correlated with SUVmax in lung AC. Zhang et al. [19] reported a significant correlation between PDL1 expression levels and SUVmax in pulmonary SCC. Takada et al. reported the association between PD-L1 expression and SUVmax in patients with small-sized lung cancer [18] or NSCLC [20], respectively. Our previous study also confirmed that SUVmax could be used to assess PD-L1 expression of PSC [22]. Similar to these
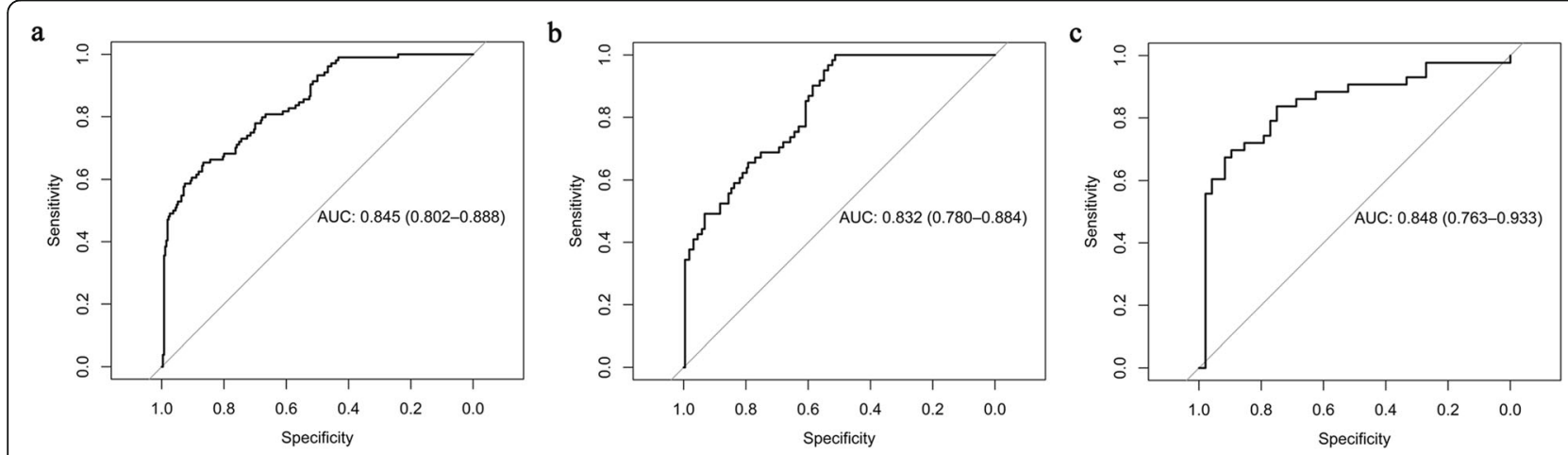

Fig. 3 Bootstrapping analysis of the ability of SUVmax to predict PD-L1 expression in overall NSCLC (a), AC (b), and SCC (c) 

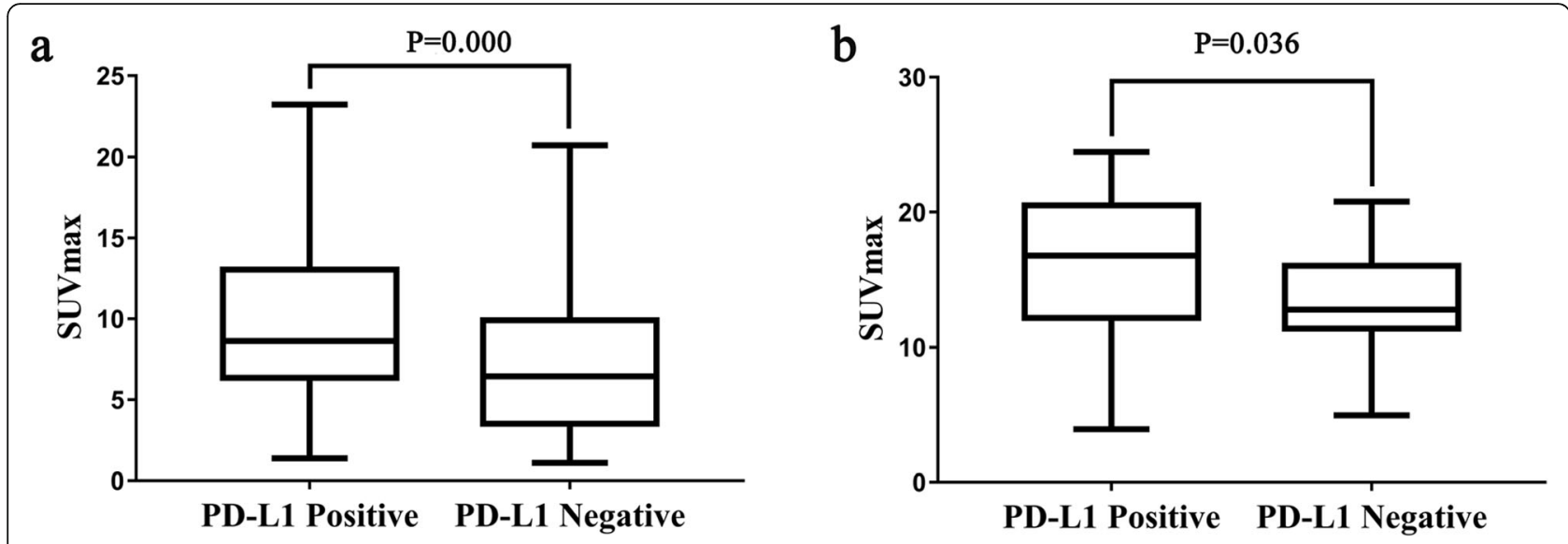

Fig. 4 The correlation between SUVmax and PD-L1 expression in AC (a) and SCC (b), respectively.

previous reports, the results of the present study showed that PD-L1 expression was associated with SUVmax in overall NSCLC, lung AC, and SCC. It may be due to altered metabolism of tumor-infiltrating $\mathrm{T}$ lymphocytes resulting from tumor-imposed glucose restriction. Chang et al. [31] showed the glucose consumption by tumors metabolically restricts $\mathrm{T}$ cells, leading to their dampened mTOR activity, glycolytic capacity, and IFN- $\gamma$ production, thereby allowing tumor progression. They also found that blocking PD-L1 directly on tumors dampens glycolysis by inhibiting mTOR activity and decreasing expression of glycolysis enzymes, reflecting a role for PD-L1 in tumor glucose utilization.

However, Jreige et al. [32] reported that no significant correlation was observed between PD-L1 tumor expression and following parameters such as SUV, MTV, and TLG in 49 cases with confirmed NSCLC. SUV represents the FDG activity of the tumor mass. MTV and TLG represent incorporating information on both tumor volume and metabolic factors. These metabolic parameters of PET/CT could reflect the glucose metabolic status of tumor tissues, which is an important feature of tumor biology [23]. Therefore, contrary to the results of Jreige et al. [32], our study found that in addition to SUVmax, TLG-P and TLG-C were also correlated with PD-L1 positivity in NSCLC. With regard to MTV, it represents the metabolic volume of the tumor mass, and different from the calculation of TLG, FDG activity is not included in the measurement of MTV. Our study showed that PD-L1 positivity had no association with the tumor metabolic volume as well as the tumor size. Thus, it demonstrated that the PD-L1-positive expression was mainly associated with FDG activity. Furthermore, when pathologically subgrouped, only SUVmax had the relationship with PD-L1 expression, which further displayed that the relationship between PD-L1 positivity and glucose activity of the tumor mass.
Moreover, this study also demonstrated that PD-L1 expression was significantly correlated with man, smoking ,and central NSCLC, which were also consistent with the previous studies [18, 20, 33]. It suggested that male, smoking-associated and central NSCLC such as lung SCC tended to exhibit PD-L1 positive expression, which confirmed the findings that PD-L1 positive rate of lung SCC was significantly higher than that of lung AC [26, 27, 29]. Furthermore, when the population of this study was pathologically subgrouped, the correlation between PD-L1 expression and these clinicopathological features disappeared, which was also in agreement with others' reports $[9,34]$. It was mainly due to that male, smoking and central tumor were major features of lung SCC, which were eliminated the interrelation with PD-L1 when compared intra-group. Moreover, the relationship between PD-L1 expression and EGFR mutation of NSCLC remains controversial. Several studies suggested that PD-L1 was highly expressed in EGFR wildtype NSCLC [35, 36]; meanwhile, a negative or no correlation between EGFR mutation and PD-L1 expression was also concluded [37-39]. For this study, almost all cases with EGFR mutation were lung ACs, and no obvious difference of PD-L1 expression in EGFR wildtype and EGER mutant tumors was found in overall NSCLC and AC groups, respectively.

Furthermore, although the results of the present study demonstrated that PD-L1 expression of NSCLC was related with SUVmax, TLG, man, smoking, and central location, multivariate analysis displayed that only SUVmax was identified as an independent predictor of PD-L1 positivity in NSCLC, which was in agreement with the previous studies $[18,20]$. It may be due to that SUVmax represents a maximally active portion of the tumor, and preferably predicts the tumor aggression. Moreover, similar to the previous reports $[19,21]$, SUVmax was also an independent and unique predictor of PD-L1 positive expression in AC and SCC subgroups, 
respectively. Subsequently, the ROC curves and the areas under the curve in this study suggested that SUVmax could predict PD-L1 expression in overall NSCLC, AC, and SCC with high sensitivity, specificity, and accuracy, respectively, which was also in line with the previous reports [18, 20, 21]. Therefore, the SUVmax of FDG PET/ CT could be helpful for exploring the existence of immune checkpoints.

Clearly, there were also several limitations in this study. Firstly, based on immunohistochemical requirements of tumor specimens for detecting PD-L1 expression, the patients included in the present study were mainly at stage I-III; therefore, cases at stage IV were quite limited, which was also the limitation of the previous studies. Secondly, due to the invasion of biopsy, the metastatic lesions were usually not biopsied again in routine clinical practice once PD-L1 expression of the primary lesion confirmed. For this study, PD-L1 expression was confirmed by the biopsy of the primary lesion. Thus, the relationship between SUVmax and PD-L1 of metastatic lesions was not analyzed. Although PD-L1 expression may exist a difference in primary and metastatic tumors, Kim et al. [40] demonstrated that the concordance of PD-L1 expression between primary and metastatic pulmonary adenocarcinomas is high when using cutoff values of $1 \%$ and $50 \%$. Thirdly, PD-L1 expression was regarded as an indicator of poor prognosis of lung cancer [41-43]. Meanwhile, SUVmax, MTV, and TLG were also reported to have the prognostic values in NSCLC [44, 45]. However, the correlations of PD-L1 expression and $\mathrm{PET/CT}$ metabolic parameters with the prognosis were not analyzed, which will be carried out in the future study. Finally, although exploring the existence of PD-L1 could provide strategies to choose immunotherapy for NSCLC, responses to PD-1/PD-L1 antibodies were also noted in NSCLC with low or absent PD-L1 expression. However, due to the small population choosing immunotherapy in this study, the correlations of PET/CT metabolic parameters and immunotherapy were not investigated. Therefore, further large-scale, prospective studies of the role of FDG PET/CT in immunotherapy are warranted.

\section{Conclusion}

PD-L1 expression of NSCLC was significantly related with SUVmax, TLG, man, smoking, and central location. No obvious difference in MTV, age, serum tumor markers, tumor size, TNM stage, and genetic mutation was observed between PD-L1 positive and negative groups. Only SUVmax of FDG PET/CT was an independent predictor of PD-L1 expression, which could help to explore the existence of immune checkpoints.

\section{Abbreviations}

NSCLC: Non-small cell lung cancer; PD-L1: Programmed cell death ligand-1; FDG: Fluorodeoxyglucose; SUVmax: Maximum standard uptake; MTV: Metabolic tumor volume; TLG: Total lesion glycolysis;

AC: Adenocarcinoma; SCC: Squamous cell carcinoma; ROls: Regions of interest; TPS: Tumor proportion score; EGFR: Epidermal growth factor receptor; KRAS: Kirsten rat sarcoma viral oncogene homolog gene; EML4ALK: Echinoderm microtubule-associated protein-like 4-anaplastic lymphoma kinase fusion gene; BRAF: V-Raf murine sarcoma viral oncogene homolog B1 proto-oncogene; ROS1: C-Ros oncogene 1; ROC: Receiver operating characteristic; CYFRA211: Cytokeratin 211; CEA: Carcinoembryonic antigen; NSE: Neuron-specific enolase; proGRP: Pro-gastrin-releasing peptide; SCCA: Squamous cell carcinoma antigen; PSC: Pulmonary sarcomatoid carcinoma

\section{Acknowledgements}

Not applicable.

\section{Authors' contributions}

$L J$ designed the study, XW collected and analyzed the data, the above two persons were major contributors in writing the manuscript. XW and $\mathrm{YL}$ analyzed and interpreted PET/CT imaging results, which were checked and proofread by Qingping Zhao. YH provided the histopathological and genetic results. LW helped the discussion. XS performed bootstrapping. All authors read and approved the final manuscript.

\section{Funding}

This work was partially supported by funds from the National Natural Science Foundation of China (81971645 and 81571703), and Outstanding Young Talents Program of Shanghai Municipal Commission of Health and Family Planning (2017YQ027).

\section{Availability of data and materials}

The datasets used and/or analyzed during the current study are available from the corresponding author on reasonable request.

\section{Ethics approval and consent to participate}

All procedures performed in studies involving human participants were approved by the ethics committee at Shanghai Pulmonary Hospital, Tongji University School of Medicine, and with the principles of the 1964

Declaration of Helsinki and its later amendments or comparable ethical standards.

\section{Consent for publication}

Not applicable.

\section{Competing interests}

The authors declare that they have no competing interests.

\section{Author details}

${ }^{1}$ Department of Nuclear Medicine, Shanghai Pulmonary Hospital, Tongji University School of Medicine, 507 Zhengmin Road, Shanghai 200433, China. ${ }^{2}$ Medical College of Soochow University, Suzhou 215123, China. ${ }^{3}$ Department of Pathology, Shanghai Pulmonary Hospital, Tongji University School of Medicine, 507 Zhengmin Road, Shanghai 200433, China. ${ }^{4}$ Department of Oncology, Shanghai Pulmonary Hospital, Tongji University School of Medicine, 507 Zhengmin Road, Shanghai 200433, China. ${ }^{5}$ Department of Thoracic Surgery, Shanghai Pulmonary Hospital, Tongji University School of Medicine, 507 Zhengmin Road, Shanghai 200433, China.

Received: 13 February 2020 Accepted: 4 May 2020

Published online: 19 May 2020

References

1. Bray F, Ferlay J, Soerjomataram I, et al. Global cancer statistics 2018: GLOBOCAN estimates of incidence and mortality worldwide for 36 cancers in 185 countries. CA Cancer J Clin. 2018;68:394-424.

2. Siegel RL, Miller KD, Jemal A. Cancer statistics, 2017. CA Cancer J Clin. 2017; 67:7-30.

3. Travis WD, Brambilla E, Nicholson AG, et al. The 2015 World Health Organization classification of lung tumors: impact of genetic, clinical and 
radiologic advances since the 2004 classification. J Thorac Oncol. 2015;10: 1243-60.

4. Travis WD, Brambilla E, Burke AP, et al. Introduction to the 2015 World Health Organization classification of tumors of the lung, pleura, thymus, and heart. J Thorac Oncol. 2015;10:1240-2.

5. Chai $T$, Zhang $P$, Lin $Y$, et al. Postoperative adjuvant therapy for resectable early non-small cell lung cancer: A protocol for a systematic review and meta-analysis. Medicine (Baltimore). 2019;98:e16468.

6. Nagasaka M, Gadgeel SM. Role of chemotherapy and targeted therapy in early-stage non-small cell lung cancer. Expert Rev Anticancer Ther. 2018;18: 63-70.

7. Garon EB, Hellmann MD, Rizvi NA, et al. Five-Year overall survival for patients with advanced nonsmall-cell lung cancer treated with pembrolizumab: Results From the Phase I KEYNOTE-001 Study. J Clin Oncol. 2019:Jco1900934.

8. Cancer Stat Facts: Lung and Bronchus Cancer. https://seer.cancer.gov/ statfacts/html/lungb.html. Accessed 31 March 2020.

9. Brody R, Zhang Y, Ballas M, et al. PD-L1 expression in advanced NSCLC: insights into risk stratification and treatment selection from a systematic literature review. Lung Cancer. 2017;112:200-15.

10. Sgambato A, Casaluce F, Sacco PC, et al. Anti PD-1 and PDL-1 immunotherapy in the treatment of advanced non- small cell lung cancer (NSCLC): a review on toxicity profile and its management. Curr Drug Saf. 2016;11:62-8.

11. Rolfo C, Caglevic C, Santarpia M, et al. Immunotherapy in NSCLC: a promising and revolutionary weapon. Adv Exp Med Biol. 2017;995:97-125.

12. Jiang M, Sun D, Guo Y, et al. Assessing PD-L1 expression level by radiomic features from PET/CT in nonsmall cell lung cancer patients: an initial result. Acad Radiol. 2019;27:171-9.

13. Jadvar H, Alavi A, Gambhir SS. 18F-FDG uptake in lung, breast, and colon cancers: molecular biology correlates and disease characterization. J Nucl Med. 2009;50:1820-7.

14. Zhou X, Chen R, Xie W, et al. Relationship between 18F-FDG accumulation and lactate dehydrogenase a expression in lung adenocarcinomas. J Nucl Med. 2014;55:1766-71.

15. Arslan E, Cermik TF, Trabulus FDC, et al. Role of 18F-FDG PET/CT in evaluating molecular subtypes and clinicopathological features of primary breast cancer. Nucl Med Commun. 2018;39:680-90.

16. Lv Z, Fan J, Xu J, et al. Value of (18)F-FDG PET/CT for predicting EGFR mutations and positive ALK expression in patients with non-small cell lung cancer: a retrospective analysis of 849 Chinese patients. Eur J Nucl Med Mol Imaging. 2018:45:735-50

17. Park HL, Yoo IR, Boo SH, et al. Does FDG PET/CT have a role in determining adjuvant chemotherapy in surgical margin-negative stage IA non-small cell lung cancer patients? J Cancer Res Clin Oncol. 2019;145:1021-6.

18. Takada K, Toyokawa G, Tagawa T, et al. Association between PD-L1 expression and metabolic activity on (18)F-FDG PET/CT in patients with small-sized lung cancer. Anticancer Res. 2017:37:7073-82.

19. Zhang M, Wang D, Sun $Q$, et al. Prognostic significance of PD-L1 expression and (18)F-FDG PET/CT in surgical pulmonary squamous cell carcinoma. Oncotarget. 2017:8:51630-40

20. Takada K, Toyokawa G, Okamoto T, et al. Metabolic characteristics of programmed cell death-ligand 1-expressing lung cancer on (18) Ffluorodeoxyglucose positron emission tomography/computed tomography. Cancer Med. 2017;6:2552-61.

21. Kaira K, Shimizu K, Kitahara S, et al. 2-Deoxy-2-[fluorine-18] fluoro-d-glucose uptake on positron emission tomography is associated with programmed death ligand-1 expression in patients with pulmonary adenocarcinoma. Eur J Cancer. 2018;101:181-90.

22. Wu X, Huang $Y$, Li $Y$, et al. (18)F-FDG PET/CT imaging in pulmonary sarcomatoid carcinoma and correlation with clinical and genetic findings. Ann Nucl Med. 2019:33:647-56.

23. Cheng $\mathrm{G}$, Huang $\mathrm{H}$. Prognostic value of (18)F-Fluorodeoxyglucose PET/ computed tomography in non-small-cell lung cancer. PET Clin. 2018;13:59-72.

24. Anwar H, Vogl TJ, Abougabal MA, et al. The value of different (18)F-FDG PET/CT baseline parameters in risk stratification of stage I surgical NSCLC patients. Ann Nucl Med. 2018;32:687-94

25. Chen R, Zhou X, Liu J, et al. Relationship between the expression of PD-1/ PD-L1 and (18)F-FDG uptake in bladder cancer. Eur J Nucl Med Mol Imaging. 2019;46:848-54.

26. Janzic U, Kern I, Janzic A, et al. PD-L1 expression in squamous-cell carcinoma and adenocarcinoma of the lung. Radiol Oncol. 2017;51:357-62.
27. Lin G, Fan X, Zhu W, et al. Prognostic significance of PD-L1 expression and tumor infiltrating lymphocyte in surgically resectable non-small cell lung cancer. Oncotarget. 2017:8:83986-94.

28. Gandhi L, Garassino MC. Pembrolizumab plus chemotherapy in lung cancer. N Engl J Med. 2018;379:e18.

29. Miyazawa T, Marushima H, Saji H, et al. PD-L1 expression in non-small-cell lung cancer including various adenocarcinoma subtypes. Ann Thorac Cardiovasc Surg. 2019;25:1-9.

30. Yang CY, Lin MW, Chang YL, et al. Programmed cell death-ligand 1 expression in surgically resected stage I pulmonary adenocarcinoma and its correlation with driver mutations and clinical outcomes. Eur J Cancer. 2014; 50:1361-9.

31. Chang CH, Qiu J, O'Sullivan D, et al. Metabolic competition in the tumor microenvironment is a driver of cancer progression. Cell. 2015;162:1229-41.

32. Jreige M, Letovanec I, Chaba K, et al. (18)F-FDG PET metabolic-tomorphological volume ratio predicts PD-L1 tumour expression and response to PD-1 blockade in non-small-cell lung cancer. Eur J Nucl Med Mol Imaging. 2019;46(9):1859-68.

33. Zhang M, Li G, Wang Y, et al. PD-L1 expression in lung cancer and its correlation with driver mutations: a meta-analysis. Sci Rep. 2017;7:10255.

34. Pan ZK, Ye F, Wu X, et al. Clinicopathological and prognostic significance of programmed cell death ligand1 (PD-L1) expression in patients with nonsmall cell lung cancer: a meta-analysis. J Thorac Dis. 2015;7:462-70.

35. Toyokawa G, Takada K, Okamoto T, et al. Relevance between programmed death ligand 1 and radiologic invasiveness in pathologic stage i lung adenocarcinoma. Ann Thorac Surg. 2017;103:1750-7.

36. Takada K, Toyokawa G, Tagawa T, et al. PD-L1 expression according to the EGFR status in primary lung adenocarcinoma. Lung Cancer. 2018;116:1-6.

37. Azuma K, Ota K, Kawahara A, et al. Association of PD-L1 overexpression with activating EGFR mutations in surgically resected nonsmall-cell lung cancer. Ann Oncol. 2014;25:1935-40.

38. D'Incecco A, Andreozzi M, Ludovini V, et al. PD-1 and PD-L1 expression in molecularly selected non-small-cell lung cancer patients. Br J Cancer. 2015; 112:95-102.

39. Hirai A, Yoneda K, Shimajiri S, et al. Prognostic impact of programmed death-ligand 1 expression in correlation with human leukocyte antigen class I expression status in stage I adenocarcinoma of the lung. J Thorac Cardiovasc Surg. 2018;155:382-92.

40. Kim S, Koh J, Kwon D, et al. Comparative analysis of PD-L1 expression between primary and metastatic pulmonary adenocarcinomas. Eur J Cancer. 2017;75:141-9

41. Mu CY, Huang JA, Chen Y, et al. High expression of PD-L1 in lung cancer may contribute to poor prognosis and tumor cells immune escape through suppressing tumor infiltrating dendritic cells maturation. Med Oncol. 2011; 28:682-8.

42. Zhou C, Che G, Zheng X, et al. Expression and clinical significance of PD-L1 and c-Myc in non-small cell lung cancer. J Cancer Res Clin Oncol. 2019;145: 2663-74.

43. Sumitomo R, Hirai T, Fujita M, et al. PD-L1 expression on tumor-infiltrating immune cells is highly associated with M2 TAM and aggressive malignant potential in patients with resected non-small cell lung cancer. Lung Cancer. 2019;136:136-44.

44. Dosani M, Yang R, McLay M, et al. Metabolic tumour volume is prognostic in patients with non-small-cell lung cancer treated with stereotactic ablative radiotherapy. Curr Oncol. 2019;26:e57-63.

45. Liu J, Dong M, Sun X, et al. Prognostic value of 18F-FDG PET/CT in surgical non-small cell lung cancer: a meta-analysis. PLoS One. 2016;11:e0146195.

\section{Publisher's Note}

Springer Nature remains neutral with regard to jurisdictional claims in published maps and institutional affiliations. 